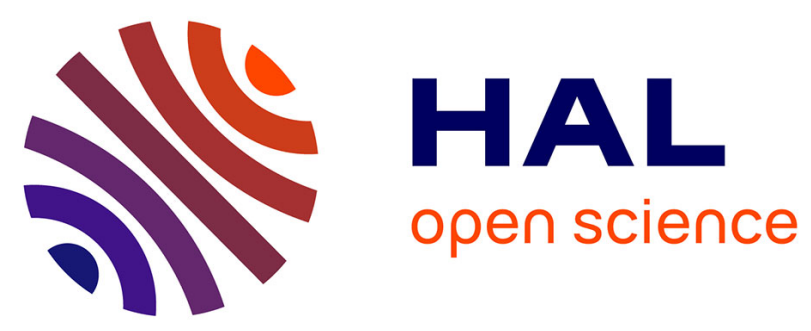

\title{
Une expertise de marché : anticipations marchandes et construction des méthodes toxicologiques dans la réglementation des produits chimiques aux États-Unis
}

David Demortain, Henri Boullier

\section{- To cite this version:}

David Demortain, Henri Boullier. Une expertise de marché : anticipations marchandes et construction des méthodes toxicologiques dans la réglementation des produits chimiques aux États-Unis. Revue française de sociologie, 2019, 60 (3), pp.429-456. 10.3917/rfs.603.0429 . hal-02463283

\section{HAL Id: hal-02463283 \\ https://hal.science/hal-02463283}

Submitted on 31 Jan 2020

HAL is a multi-disciplinary open access archive for the deposit and dissemination of scientific research documents, whether they are published or not. The documents may come from teaching and research institutions in France or abroad, or from public or private research centers.
L'archive ouverte pluridisciplinaire HAL, est destinée au dépôt et à la diffusion de documents scientifiques de niveau recherche, publiés ou non, émanant des établissements d'enseignement et de recherche français ou étrangers, des laboratoires publics ou privés. 


\section{Une expertise de marché : anticipations marchandes et construction des méthodes toxicologiques dans la réglementation des produits chimiques aux États-Unis}

David Demortain, directeur de recherche INRA, Laboratoire Interdisciplinaire Sciences Innovations Sociétés (LISIS), david.demortain@inra.fr

Henri Boullier, post-doctorant IFRIS, Centre de recherche médecine, sciences, santé, santé mentale, société (Cermes3)

A paraitre dans la Revue Française de Sociologie. Pour citer l'article : Demortain, D., \& Boullier, H. (2019). « Une expertise de marché : Anticipations marchandes et construction des méthodes toxicologiques dans la réglementation des produits chimiques aux États-Unis », Revue Française de Sociologie, Vol. 60(3), 429-456. 


\section{Résumé}

L'évaluation de produits est souvent un préalable à leur mise sur le marché. Dans le cadre de politiques sanitaires et environnementales, elle s'appuie sur une expertise réglementaire régulièrement accusée de privilégier les intérêts des industries assujetties, du fait de leur emprise sur l'économie politique de la science. À partir de l'histoire d'une méthode d'évaluation des produits chimiques promue par l'Environmental Protection Agency (EPA) à partir des années 1980, la modélisation des relations structure-activité, cet article examine le rôle respectif des industries et des administrations dans la conception, l'utilisation et la standardisation de l'expertise scientifique des produits. La structuration progressive du travail d'évaluation de l'EPA autour des relations structure-activité, une approche jusque-là plutôt mobilisée par l'industrie pour le développement de nouvelles molécules, montre que cette expertise est le fruit d'une série d'anticipations croisées des industriels et de l'administration en charge de sa réglementation, concernant le marché en train de se former et la somme des biens, incertains, qui le constitue. Loin de manifester une capture des agences par les industries, le cas étudié ici montre comment les interactions entre une administration, des scientifiques et des entreprises ont participé au développement d'une expertise de marché. 


\section{Introduction $^{1}$}

Les règlementations industrielles imposent fréquemment l'évaluation ou le test des produits avant leur mise sur le marché. Cet instrument dit de «gate keeping 》(Carpenter 2010, Faulkner 2017) semble, comme d'autres, soumis à l'influence des industries assujetties à cette intervention publique. Comme le scandale Volkswagen le montre, ainsi qu'une série d'affaires touchant, par exemple, à des produits pharmaceutiques, les industriels sont largement représentés dans les organismes qui conçoivent les critères d'évaluation des produits, les seuils de risque à appliquer, et les tests qui sont sensés pouvoir les documenter. Les industries concernées conduisent souvent ces tests elles-mêmes, et contrôlent la présentation et l'interprétation de leurs résultats, grâce à l'établissement de relations serrées avec les autorités d'évaluation et leurs experts.

Le processus d'évaluation des produits et la science qui est mobilisée dans ce processus-dite science réglementaire (Borraz et Demortain 2015, Demortain 2017)-pourrait ainsi traduire une influence industrielle plutôt qu'une capacité bureaucratique d'intervention sur les marchés. La science réglementaire est d'ailleurs souvent visée dans la dénonciation aujourd'hui de plus en plus fréquente de la « capture » des agences de régulation (Carpenter et Moss 2014), des conflits d'intérêt dans lesquels se trouveraient les scientifiques officiant dans ces procédures et, plus généralement, l'influence des industries réglementées sur l'économie politique de la science (Hauray, 2015, Krimsky, 2004 ; Lave et al., 2010). Une science réglementaire comme la toxicologie serait un terrain de lutte favorable aux intérêts industriels (Boullier 2016a, 2019; Henry 2017). Elle est aujourd'hui appropriée par des acteurs qui

\footnotetext{
Cet article s'appuie sur des enquêtes conduites dans le cadre du projet ANR INNOX (« L'innovation dans l'expertise : la modélisation et la simulation comme modes d'action publique », INOV-13-SOIN-005), entre 2014 et 2017. Nous remercions tout particulièrement Matthieu Ansaloni pour sa relecture d'une version antérieure de l'article, ainsi que les évaluateurs anonymes de la revue pour leurs commentaires et leurs suggestions d'amélioration de l'article.
} 
insistent sur son caractère innovant (Moghissi et al., 2014), montrant qu'elle intègre les développements les plus actuels des disciplines correspondantes comme la biologie ou l'informatique-conduisant à la molécularisation (Judson et al., 2018) ou au «tournant computationnel » (Varenne et Silberstein, 2013). Ces innovations pourraient renforcer encore davantage le pouvoir de fait des industriels, puisqu'elles élèvent le niveau de complexité des méthodes et de preuve scientifique requis pour établir la dangerosité d'un produit et en justifier le retrait. Seules les industries dotées de l'expertise scientifique la plus poussée parviennent à faire la preuve des qualités de leurs produits, influençant ainsi les décisions à leur avantage.

Cet article cherche à comprendre dans quelle mesure l'expertise réglementaire, au sens de la science employée par les administrations ayant des pouvoirs de réglementation des industries, reflète effectivement l'influence des industries, ou révèle au contraire une capacité administrative d'intervention sur les marchés. La question continue de se poser au regard des recherches récentes, qui montrent que les autorités publiques conservent un pouvoir non négligeable d'organisation du marché : les relations étroites qu'elles établissent avec les représentants des industries leurs permettent de les structurer, notamment en diffusant des critères et des normes d'évaluation, qui contribuent à hiérarchiser les entreprises et les produits (Dubuisson-Quellier 2017). Les autorités peuvent aussi mobiliser les règles de fonctionnement typiques des marchés, comme la mise en concurrence, pour regagner une capacité à intervenir dans des secteurs dont elles étaient jusqu'à présents absentes (Ansaloni et Smith 2017). Les normes d'évaluation des produits qu'elles appliquent façonnent les entreprises, leurs expertises et modes de développement de produits (Benoit 2018, Carpenter 2010, Wengle 2016).

La question du pouvoir relatif des industries et de l'administration dans la construction d'un marché reste ainsi ouverte, les cas examinés dans la littérature étant trop variés et épars pour 
produire des conclusions générales. Il n'est pas certain, non plus, que faire ou organiser des marchés ne traduise pas, précisément, une réduction de la capacité de l'État à imaginer et imposer des objectifs d'action publique. De quelle capacité d'action publique parle-t-on quand cette capacité est strictement réduite à l'organisation, l'extension ou l'imitation de ce qu'est un marché supposé ? Il reste, enfin, un travail à opérer sur la conceptualisation de cette notion de capacité d'intervention, souvent réifiée, indexée sur des ressources ou des instruments fixes, plutôt que construites dans le cours des interactions avec les acteurs industriels et les capacités qu'eux-mêmes expriment dans leur travail, simultané, de façonnage d'un marché. Cet article prolonge la discussion des pouvoirs et capacités relatives de l'administration et de l'industrie, en prenant comme entrée les savoirs sur lesquels reposent l'évaluation publique des produits industriels. Empiriquement, il examine l'histoire d'une méthode de production et d'interprétation de données toxicologiques, et pose la question de savoir quelle est la part propre de l'administration et de l'industrie dans son développement.

Dans le cadre du contrôle de la mise sur le marché des substances chimiques, les entreprises soumettent aux autorités des données sur la toxicité de la molécule qu'ils souhaitent commercialiser. Elles peuvent être produites à partir de plusieurs méthodes : des méthodes animales, dites in vivo, selon lesquelles on teste la substance sur une espèce donnée, à une dose donnée, pour révéler les points de l'organisme animal affectés. Des données du même ordre peuvent être produites in vitro, à partir de dispositifs de test sur des matériaux biologiques notamment cellulaires. Une troisième méthode consiste à extrapoler les résultats de données in vivo ou in vitro existantes, pour «prédire» la toxicité de substances sur lesquelles on ne dispose pas de telles données. Cette approche, dite in silico, prend souvent la forme de «structure-activity relationships », du nom de la théorie chimique qui valide cette 
extrapolation de résultats expérimentaux concernant une substance, à une autre substance². L'extrapolation est jugée possible si les deux substances en question ont une structure moléculaire semblable. La méthode structure-activité peut être quantitative (on emploie alors des modèles mathématiques qui résume la relation statistiquement établie entre un indicateur structurel $\mathrm{x}$, telle la solubilité de la substance, et une forme de toxicité $\mathrm{y}^{3}$ ) ou qualitative : c'est le cas lorsqu'un chimiste ou un toxicologue convoque sa connaissance de la structure et de la toxicité typique d'une famille de molécules, et juge qu'une substance nouvelle appartient à cette famille - et est donc susceptible de manifester la même toxicité. Cette méthode structure-activité, qui relève de la toxicologie computationnelle, est notamment utile lorsque l'on n'a pas la capacité ou les ressources de mener des expérimentations in vivo ou in vitro spécifiques sur une nouvelle substance. Elle a été conçue par l'Environmental Protection Agency (EPA) américaine à la fin des années 1970 pour évaluer rapidement les risques de toxicité des substances et repérer les plus problématiques au moment de l'examen des nombreuses demandes de mise sur le marché—en un terme anglais, pour «screener ». L'EPA a depuis été suivie par de nombreuses autres agences à travers le monde.

L'emploi d'une expertise spécialisée sur les relations structure-activité semble être à première vue le produit de l'influence de l'industrie chimique américaine : celle-ci a imposé des délais extrêmement courts d'évaluation des produits chimiques, évité d'avoir à produire des données expérimentales, et placé la charge de la preuve de la dangerosité des substances chimiques sur l'agence (Boullier 2016b, Creager 2018, Demortain 2020, Vogel et Roberts 2011). Elle a en

Cette méthode est promue par l'Agence européenne des produits chimiques et par l'Organisation de Coopération et de Développement Économique (OCDE 2006, ECHA 2017). Elle prend le nom dans ce contexte de « read-across » ou « lecture croisée ».

${ }^{3}$ Pour produire une estimation de la toxicité d'une nouvelle molécule, on applique à ses descripteurs une équation mathématique formulant la relation statistique entre un paramètre moléculaire et un ensemble de données de toxicité obtenues par des expériences sur animaux d'une série de substances relevant d'une même famille. La qualité de la prédiction dépend de la robustesse de ces données initiales ayant servi à formuler la relation mathématique, et de l'appartenance de la nouvelle molécule à une famille de molécules pour laquelle ladite équation est valide (une substance dite co-générique). 
outre rendu difficile la mise en œuvre de la réglementation, en soumettant à l'EPA des centaines de produits à évaluer chaque semaine. Dans ces conditions, l'EPA n'avait visiblement d'autre choix que de faire de l'évaluation des relations structure-activité, seule méthode pour screener rapidement et de manière crédible les produits sans passer par des tests sur animaux, qu'elle ne pouvait pas conduire elle-même et que la loi ne lui permettait pas de requérir. L’influence de l'industrie est symbolisée, enfin, par le fait que la méthode structureactivité a été initiée par l'industrie pharmaceutique, et est donc une expertise industrielle. Cet article montre, toutefois, que l'agence n'a pas fait que recourir à un instrument d'évaluation préconçu pour s'adapter aux contraintes créées par les stratégies industrielles. Elle a plutôt activement conçu les connaissances de type structure-activité et leur usage, en fonction des effets que cette méthode promettait d'obtenir sur les firmes et le marché en formation.

En rentrant dans le processus de détermination des contenus et en analysant les effets de la méthode structure-activité, cet article montre que les positionnements de l'administration et de l'industrie vis-à-vis de cette science se sont construits au cours du temps, dans le cours d'une interaction relativement labile entre spécialistes travaillant pour l'administration, experts industriels et universitaires. Les méthodes utilisées par l'EPA sont endogènes aux relations entre les acteurs parties prenantes de la réglementation de cette industrie et de cet ensemble émergent de produits. Leur usage reflète la succession de leurs calculs croisés quant aux effets de l'application d'un type de savoir prédictif sur le marché en train de se dessiner. Si l'un ou l'autre acteur a un pouvoir, ce n'est pas un pouvoir structurel, mais un pouvoir incrémental sur la production des savoirs et des outils réglementaires. De ce point de vue, le pouvoir des fonctionnaires experts de l'agence environnementale américaine est loin d'être négligeable, même si l'application de la méthode des relations structure-activité se traduit finalement par la mise sur le marché de la majorité des produits. 
Pour mener à bien cette analyse, nous avons conduit quatorze entretiens avec dix informateurs différents, fonctionnaires du bureau des substances toxiques ou chercheurs de l'Office of Research and Development de 1'EPA, passés ou présents ${ }^{4}$. Les entretiens étaient de type histoire orale, et couvraient les activités du bureau, ses procédures de décision, l'utilisation des méthodes structure-activité, les programmes de recherche et de production de données menés, la structure des relations internes et externes du bureau (avec le département de recherche, avec le syndicat industriel et les entreprises individuelles notamment), et ont duré entre 35 minutes et deux heures. Deux informateurs, le directeur de la «structure-activity team » et le directeur de la Environmental Effects Branch supervisant le groupe de spécialistes des relations structure-activité, nous ont accordé plusieurs entretiens, et continué de partager des éléments d'information et d'analyse à travers une correspondance électronique. Nous avons également mobilisé trois entretiens conduits par des chercheurs de la Chemical Heritage Foundation dans le cadre de son TSCA Oral History Project. Dans un contexte où beaucoup d'acteurs de l'époque ont disparu, dans la mesure où une grande partie des événements se sont déroulées dans les années 1970 et 1980, ces sources nous ont fourni des données précieuses pour préciser certaines de nos hypothèses et consolider nos analyses. Nous avons ensuite mobilisé une quarantaine de documents issus du fonds d'archives d'un ancien fonctionnaire de l'agence et des archives électroniques de l'EPAs. Elle s'appuie, enfin, sur une connaissance de fond de l'histoire, des programmes et des acteurs de l'EPA gagnée au cours de travaux parallèles (Boullier 2016c, Demortain 2017).

\footnotetext{
“ Dans la suite du texte, le terme de «fonctionnaires de l'agence » renvoie aux membres du bureau des substances chimiques, qui ont été nos principaux informateurs, tandis que l'«agence» désigne cette administration plus généralement.

s Les documents exploités incluent des rapports d'activité du bureau des substances chimiques (OTS), chargé de mettre en œuvre la réglementation des substances chimiques industrielles, des rapports d'évaluation de la politique des produits chimiques, des rapports techniques sur les tests des produits chimiques et les méthodes de modélisation et des présentations à des conférences et des publications scientifiques transmises par les informateurs.
} 
Dans un premier temps, l'article justifie l'intérêt d'entrer dans la question des relations entre administration et industrie et de l'organisation des marchés par l'expertise, puis développe l'histoire de l'utilisation du SAR à l'EPA, montrant comment la décision d'y recourir et la définition de la méthode reflètent les ajustements entre industriels et agences, du point de vue des effets qu'ils souhaitent obtenir, à travers cette méthode, sur le marché. La troisième partie développe les implications de ce cas et de cette approche de l'expertise scientifique.

\section{L'expertise scientifique face aux industries et la construction des marchés}

La littérature disponible sur l'expertise scientifique à finalité politique (Granjou 2004, Joly 2005) a très abondamment étudié les interactions entre acteurs relevant de la science et les acteurs administratifs ou politiques, ainsi que les contextes institutionnels nationaux et configurations de controverse dans lesquels ces interactions se déroulent (Joly 2016). Elle fait de ces interactions un facteur théorique important pour expliquer quelle connaissance est reconnue comme expertise dans nos sociétés. La recherche disponible s'ajuste à ce que les décideurs considèrent comme crédible pour constituer une décision légitime dans une configuration politique donnée. Un jeu se noue entre les scientifiques, qui tendent à purifier leurs connaissances pour la faire apparaitre comme objective, proche des canons de la science, et les décideurs cherchant une connaissance en appui à une politique publique donnée. S'en suit un jeu autour de la frontière entre science et politique (Joly 2012) qui se stabilise autour de conventions de production et d'interprétation des connaissances, démarquant par exemple un territoire scientifique d'évaluation des risques et un territoire plus politique de gestion des risques (Demortain 2020 ; Jasanoff 1987, 1990).

Dans cette perspective, l'expertise dominante (les exemples les plus fréquemment cités sont les modèles climatiques, ou les tests toxicologiques) est celle qui se construit dans le réseau 
des relations durables tissées entre un groupe de scientifiques et des acteurs institutionnels. Le résultat est une forme de coalition épistémique dans laquelle des acteurs différents fonctionnaires, personnel politique, universitaires, etc. - préservent de concert les méthodes et les résultats qui confirment les options politiques choisies, et permettent aux scientifiquesexperts de gagner une autorité liée à la reconnaissance de leurs savoirs par une organisation gouvernementale (Irwin et al. 1997 ; Shackley et Wynne 1996,). Ce genre d'analyse a été systématisé depuis dans les termes d'une approche dite co-productionniste (Jasanoff 2004), selon laquelle l'expertise scientifique mobilisée par les administrations publiques pour la réglementation des industries, la science réglementaire (Demortain 2017 ; Jasanoff 1990), serait le produit d'une double-détermination, par ce qui est considéré comme une science crédible et objective d'une part, et ce qui est requis comme standard juridique de preuve d'autre part.

L'insistance sur ces deux déterminants (science et politique publique, ou science et droit selon les auteurs) fait que les organisations et connaissances industrielles, pourtant centrales lorsque l'action publique relève de la réglementation de la production et de la consommation de biens, ont été relativement négligées dans l'étude de l'expertise scientifique (Frickel et Moore, 2006). Cette dimension est cependant progressivement réinvestie, notamment par la littérature sur la production de l'ignorance, le lobbying et l'économie politique de la science (Frickel et Vincent 2007, Michaels 2008, Oreskes et Conway 2010). La notion de capture des autorités par les industries par le biais de la science que ces dernières produisent et valorisent, constitue la base des discussions dans ce champ. Elle renvoie à la capacité des industries à façonner un contexte de connaissance et des réseaux scientifiques, dont les autorités publiques ne peuvent se déprendre. Si cette idée de capture de l'État par les intérêts privés est indéniablement évocatrice et fertile, notamment parce qu'elle force à prendre au sérieux la crédibilité et la capacité d'expertise de l'industrie, son défaut est double : elle approche les acteurs concernés 
de manière générique et monolithique, en leur assignant un intérêt ou un objectif ; elle tend à réifier les connaissances mobilisées comme si elles constituaient une ressource ou une capacité prédéfinie. Ce faisant, elle réduit largement la part des interactions entre les acteurs industriels, gouvernementaux, scientifiques et autres dans la composition des connaissances, des cadres cognitifs crédibles, pour conforter une vision in fine plutôt structurelle que relationnelle du pouvoir (Strange 2015). La construction des capacités de l'État de piloter, d'orienter, organiser les interactions entre acteurs-dont la littérature montre qu'elles sont centrales dans les nouveaux modes d'intervention publique (Dubuisson-Quellier 2017 ; Gensch et Zangel 2011 ; King et Le Galès 2011) - reste alors à comprendre.

Le point de départ de la démarche proposée ici pour dépasser le diagnostic de capture, consiste à redéfinir l'expertise scientifique comme un travail d'évaluation constitutif du marché : une forme de régulation par l'évaluation des qualités incertaines des biens, reposant sur l'emploi de données, de méthodes et de protocoles permettant de comparer ces biens. Il s'agit en d'autres termes d'une régulation à la fois industrielle et administrative, puisque ces données, méthodes et protocoles sont négociés par les acteurs engagés dans la définition des biens qui vont être échangés sur ce marché. L'évaluation scientifique de produits aux effets incertains, des médicaments aux produits chimiques, en passant par les organismes génétiquement modifiés ou les nanotechnologies (Boudia et Demortain 2014, 2015), est bien un processus de construction d'un marché, puisque de ces règles dépendent la définition des qualités d'un produit et le calcul de sa valeur (Callon et al. 2000, Callon et al. 2002). L'évaluation implique la mesure de ce que vaut le produit, sur des échelles conventionnelles et formelles de prix, de risque ou de bénéfice pour la santé. L'évaluation des produits et des risques, précisément, cherche à lever les incertitudes sur les qualités des biens, construisant des indications sur lesquelles s'appuie la mise en marché. Elle le fait sur la base de tentatives de prédiction des effets futurs, des incertitudes qui concernent l'objet, à partir d'indicateurs 
spécifiques ou de «proxies» (Knorr-Cetina 2012). L'évaluation réglementaire des produits chimiques prend fréquemment la forme de mise en liste (Boullier 2016c) ou de classement d'un nouvel objet par rapport à d'autres objets connus, ou par référence à un niveau de dangerosité ou de bénéfice normal (au sens statistique), ressortant de données historiques (Faulkner 2012). Ces expertises du chimique sont particulières : elles servent à qualifier un bien particulier, mais nécessitent une connaissance ou une anticipation de la somme des autres biens, substances, avec lesquels ils sont susceptibles d'être associés. La chimie est une science inventive, qui procède par recombinaison constante pour inventer de nouvelles entités (Barry 2005 ; Bensaude-Vincent et Stengers 2001). La chimie et l'évaluation de ses productions est marquée par l'incertitude, puisque l'espace de ces combinaisons - «l'espace chimique », dans les termes des chimistes - et les combinaisons possibles sont potentiellement infinis.

Étant donné que l'expertise scientifique participe de la construction d'un marché, elle ne peut pas être considérée comme extérieure à celui-ci, ou plus précisément extérieure aux interactions entre les acteurs dont l'enjeu commun est la définition, ou la levée des incertitudes sur les biens qui viendront à être échangés. Dans un sens, les tests sont un mécanisme marchand autonome de classification des qualités des produits. Ils sont pratiqués dans le cadre de la R\&D des entreprises, définis dans des consortiums de standardisation souvent privés. La capacité à définir un test approprié est au principe de la réussite d'une entreprise et de ses innovations, et donc un modulateur important de la concurrence sur les marchés. Dans un autre sens, les tests sur les produits sont pour partie au moins conçus, vérifiés et leurs résultats interprétés par des experts scientifiques qui travaillent pour l'administration. À travers cette activité, ils dictent à l'industrie les critères de qualité d'un bien considéré comme sûr.

En descendant dans le détail de la construction de cette expertise, de la définition des qualités incertaines des objets évalués, de la somme de ces objets, et des connaissances nécessaires 
pour lever cette incertitude, apparaissent des mécanismes et des degrés d'influence mutuelle entre les acteurs administratifs, scientifiques et industriels, qui permettent ainsi de relativiser la situation de capture. De même que l'évaluation des produits est une affaire de calculs pour prédire les propriétés des produits, l'évaluation des produits fait elle-même l'objet d'anticipations marchandes de la part des industriels, des scientifiques ou des fonctionnaires de l'agence de régulation. En s'appuyant sur Beckert $(2012,2016)$, on peut définir ces anticipations marchandes comme des attentes, d'ordre fictionnelles ou imaginaires, concernant les biens pouvant circuler sur un marché incertain, en formation ${ }^{6}$. Ces anticipations marchandes ont trois dimensions: elles sont marquées par l'incertitude entourant les propriétés de ces biens, ainsi que leur nombre et leur diversité même dans le cas des produits chimiques. Elles sont donc construites par référence à l'expérience des interactions avec les acteurs dont dépend cette qualification, et émergent du réseau des relations qui les lient et de leurs interactions répétées. Elles influencent les actions des acteurs sur les règles d'évaluation des produits. L'évaluation scientifique prend forme dans la suite des anticipations que les acteurs font sur la qualité et la quantité des biens composant le marché : quelle proportion de produits, à l'aune d'un mode d'évaluation donné, va apparaitre comme dangereuse ? Le mode d'évaluation scientifique des produits est-il susceptible de créer des faux négatifs ou des faux positifs, et si oui en quelle proportion? De quels produits peut-on prévoir la commercialisation? Quelle est la taille du marché que dessinera l'accumulation de ces évaluations et de ces décisions ? Les tests appliqués et la connaissance scientifique disponible sont-ils suffisamment crédibles pour que les décisions soient acceptées, et que

\footnotetext{
- Beckert forge l'expression d'anticipations fictionnelles pour remplacer celle d'attente rationnelle, qui domine la théorie économique. Il y inclut l'ensemble des images et perceptions que les acteurs forment lorsqu'ils anticipent un état du monde et du marché, et considèrent les actions qu'ils peuvent conduire pour influencer cet état. Il dépeint cette activité de construction d'anticipations imaginaires comme aussi générique que celle de calcul rationnel, mais devant la remplacer car plus juste empiriquement. Il y inclut également, ce qui est clé pour notre propos, «les qualités symboliques que les acteurs attribuent aux biens et qui transcendent les propriétés matérielles de ces biens » (Beckert 2016, p. 9, nous traduisons).
} 
l'administration en charge parvienne à produire les effets sur l'industrie qu'elle cherche à avoir?

L'hypothèse qui est faite ici est que l'évaluation scientifique des produits, et le type d'expertise scientifique mobilisé par l'agence réglementaire, prend forme au fur et à mesure que ces anticipations par les acteurs parties prenantes de la régulation de cette industrie, se déploient et croisent. Ce réseau d'anticipations présiderait à la définition des capacités à acquérir pour intervenir sur les produits. Dans un contexte où une incertitude entoure la somme des produits qui vont être développés et proposés à la commercialisation par les industries, et la capacité à obtenir de l'information sur ceux-ci pour les qualifier, il pointerait ainsi vers une expertise comparative, employant des informations existantes pour extrapoler des biens connus, au nouveau produit - précisément ce qu'est la science des relations structure-activité. L'expertise ne serait donc pas exogène par rapport au marché, puisque l'agence et ses scientifiques eux-mêmes produisent leurs calculs en fonction de la situation qu'ils ont à gérer, et des signaux qu'ils reçoivent de l'industrie. L'histoire de l'application des méthodes computationnelles fondées sur la théorie structure-activité, à l'EPA, porte la trace de cet ajustement dynamique et indirect entre les acteurs du marché sur les qualités génériques de ces produits, comme leur toxicité, et les manières de les mesurer.

\section{L'EPA, le marché des produits chimiques et le recours au QSAR}

La modélisation des relations structure-activité est apparue comme un instrument d'évaluation des produits chimiques réglementés dans un contexte historique bien particulier : celui de la négociation d'une série de lois environnementales ambitieuses dans les années 1960 et 1970, largement combattues par l'industrie chimique et pétrolière, qui n'aura de cesse d'en limiter la portée. 
Formuler le manque de données pendant les négociations du TSCA (1973-1979) : l'intérêt naissant de l'EPA pour les relations structure-activité

Dans les années 1960, le mouvement environnementaliste naissant et des scandales comme la marée noire de Santa Barbara en 1969 emmenèrent les politiques sur des terrains qu'ils avaient jusque-là peu explorés. Le contrôle des effets des substances chimiques sur l'homme et l'environnement devint une priorité pour le candidat Nixon. Peu de temps après sa prise de fonction en 1969, et pour contrer la popularité du sénateur démocrate Muskie, champion de la défense de l'environnement, Nixon suivit les recommandations de quelques-uns de ses conseillers de mettre en place une agence - la future EPA - et une loi sur les substances chimiques toxiques (Boullier, 2019 ; Davies et Davies 1970). L'EPA vit le jour en décembre 1970 , tandis que débutait la négociation d'un texte de loi encadrant la commercialisation des substances chimiques, le Toxic Substances Control Act (TSCA). Le texte, parallèlement à de nombreux autres nouveaux textes de loi sur la qualité de l'air, de l'eau, des risques des pesticides, des effets des produits chimiques sur la santé au travail, fait partie de la vague de la social ou risk regulation, de philosophie précautionniste, et que l'industrie chimique va largement s'employer à contenir (Vogel 1988).

Dès le départ, l'écriture du projet de loi s'avéra difficile. La première version du texte s'articulait autour de deux principes, calqués sur ce qui faisait dans le domaine des pesticides et dans le domaine du médicament ${ }^{8}$ : un dispositif de demande de mise sur le marché d'une part, dans lequel la charge de la preuve de l'innocuité du produit repose sur l'entreprise pétitionnaire. Ces principes firent rapidement l'objet de négociations ardues, notamment parce que le texte couvrait un vaste territoire de nouveaux produits, aux propriétés et aux

\footnotetext{
Richard Nixon, Special Message to the Congress about Reorganization Plans to Establish the Environmental Protection Agency and the National Oceanic and Atmospheric Administration, 9 juillet 1970, p. 578-586, [en ligne] <http://quod.lib.umich.edu/p/ppotpus/4731750.1970.001> (page consultée le 15 janvier 2019)

${ }^{8}$ Respectivement encadrés par le Federal Insecticide, Fungicide, and Rodenticide Act de 1910 et le Federal Food, Drug, and Cosmetic Act de 1938.
} 
applications mal connues (l'approche juridique employée jusqu'ici consistait à réglementer la présence de produits dans l'eau, dans l'air, dans les sols, etc.). Terry Davies, conseiller à la Maison Blanche, auteur et promoteur de cette régulation transversale de la chimie, dut engager une négociation du texte avec James Lynn, avocat du département du Commerce et porteur, pour l'occasion, des préoccupations et réticences de l'industrie chimique vis-à-vis de ce texte, dont elle pense qu'il va bloquer la commercialisation de nouvelles substances. Lynn obtint que la charge de la preuve soit renversée : ce serait à l'EPA de faire la preuve de la dangerosité du produit pour pouvoir stopper sa mise sur le marché (Boullier, 2019).

Partiellement vidée de sa substance, la proposition de Toxic Act fut ensuite discutée au Congrès, où elle disposait de peu de défenseurs. Perçue comme pro-industrie suite aux concessions obtenues par le département du Commerce, elle fut boudée par l'Environment and Public Works Committee ${ }^{9}$, un comité qui est habituellement un allié précieux des réformes sanitaires et environnementales et qui a précédemment soutenu l'adoption de textes importants comme le Clean Air Act de 1963 et le Safe Drinking Water Act de 1974. L'industrie chimique était au contraire activement impliquée dans la négociation du texte. C. Boyd Shaffer, pour le compte de la Manufacturing Chemists Association (la principale association industrielle du secteur), parvint à bloquer un amendement requérant des tests toxicologiques préliminaires pour les nouveaux produits chimiques, cela risquant de drastiquement limiter la capacité d'innovation des plus petites sociétés du secteur. Le juriste de l'association prit part à la rédaction du texte et y inscrivit le compromis établi entre l'industrie, le Congrès et le CEQ : l'industrie acceptait qu'un cadre réglementaire spécifique pour les nouveaux produits chimiques soient établies, pour peu qu'aucune obligation de test préalable ne soit imposée (Craeger 2018). 
Les discussions au Congrès poussèrent assez tôt les fonctionnaires de l'EPA à réfléchir à des moyens d'évaluer les produits chimiques sans test toxicologique. L'intérêt pour la modélisation des relations structure-activité a en effet émergé pendant ces années de négociation de la loi, qui laissent à l'agence le loisir d'anticiper le travail difficile qu'elle aurait à faire pour la mettre en œuvre. Le bureau des substances chimiques (OTS), inauguré en 1974, se rend compte qu'il va devoir évaluer un grand nombre de données sur des milliers de substances chimiques nouvelles, aux propriétés encore inconnues. Pendant cette période préparatoire, les fonctionnaires de l'agence se mettent en relation avec des organisations scientifiques comme le National Institute of Environmental Health Sciences et la National Science Foundation, pour réfléchir aux méthodes possibles. Ces échanges les orientent vers l'idée, venant de la chimie computationnelle, selon laquelle certaines propriétés génériques des substances, et donc certaines formes de toxicité, peuvent être déduites de leur structure moléculaire. C'est dès 1973 que les fonctionnaires du futur OTS avaient identifié un corpus de littérature significatif sur les corrélations et les méthodologies structure activité.

La modélisation des relations entre des propriétés chimiques et des impacts biologiques est ancienne, et relève de la chimie fondamentale ${ }^{10}$. L'approche quantitative de cette corrélation est, elle, alors encore toute récente. Elle fut initiée par un professeur de chimie à Pomona College en Californie, Corwin Herman Hansch (1918-2011), connu comme le père du design de molécule assisté par ordinateur. Les « équations de Hansch »" résument les relations entre structure et activité, au cœur des modèles QSAR, permettent de produire une estimation

\footnotetext{
${ }^{10} \mathrm{D}$ 'après la troisième édition de l'Encyclopedia of Toxicology, elle remonterait à 1860, quand la relation entre activité physiologique et structure moléculaire a été exprimée pour la première fois avec la formule $\Phi=\mathrm{f}(\mathrm{C})$ par Crum-Brown et Fraser (1868). Quelques années plus tard, Meyer et Overton (1899) ont découvert qu'il y avait une corrélation entre l'hydrophobie d'une substance (le fait qu'elle ne se mélange pas avec l'eau) et son potentiel narcotique.

"Il a développé ces équations avec d'autres chimistes, et ils ont ensuite promu leur utilisation dans l'industrie pharmaceutique pendant les années 1970 et 1980, conduisant à une situation où les départements de R\&D des firmes pharmaceutiques possèdent systématiquement des unités de computational drug design (conception numérique de molécules) qui collaborent avec les toxicologues qui conduisent les premiers tests de toxicité sur les animaux.
} 
numérique de toxicité. Les outils qui mobilisent la théorie structure-activité permettent de déduire qu'une molécule qui est similaire à une classe de substances pour laquelle la formule a été développée, générera le même niveau de toxicitén

La structure moléculaire et les descripteurs de base font partie des données dont l'EPA, au vu de la négociation en cours, pense qu'elle pourra bénéficier, malgré le peu de données toxicologiques demandées à l'industrie. À la lumière de la théorie structure-activité, il apparait donc à l'agence qu'elle pourra à minima passer en revue les nouvelles substances (faire un «screening ») et formuler rapidement des hypothèses quant à leur toxicité. Au milieu des années 1970, l'agence signe plusieurs contrats avec des instituts de recherche privés pour explorer cette idée. Le Franklin Institute Research Laboratories développe alors une bibliographie sur les corrélations structure activité (FRI 1975), et des essais d'application de méthodologies pour prédire la toxicité de substances organiques (FRI 1976).

En 1976, après six ans de discussions, la loi fut promulguée par le Président Ford. L'industrie avait obtenu des obligations de test très restreintes. Pour les substances déjà présentes sur le marché, le texte ne donne pas les moyens à l'EPA de formuler des interdictions fermes ${ }^{13}$ (Vogel et Roberts, 2011). Pour les substances considérées comme nouvelles (encore jamais commercialisées, au regard du registre des substances existantes compilé par le bureau), le texte impose à l'EPA de dire en moins de 90 jours s'il existe une suspicion raisonnable d'un danger pour la santé humaine, pour aller à l'encontre de la mise sur le marché. La commercialisation est l'issue par défaut de ce délai. À l'EPA de bloquer cette issue, et de faire

\footnotetext{
12 Quand ils parlent d' « outils»SAR ou QSAR, les acteurs qui les mobilisent font référence à différentes ressources matérielles qui permettre de produire cette formule: les bases de données qui contiennent des résultats expérimentaux sur lesquelles des régressions statistiques peuvent être conduites, les techniques statistiques elles-mêmes, ou encore les terminologies et descripteurs harmonisés qui permettent de former des catégories de substances chimiques.

${ }_{13}$ Seulement cinq interdictions ont été prononcées depuis son adoption en 1976. Il s'agit des PCB, des chlorofluorocarbones, de la dioxine, de l'amiante et du chrome hexavalent. Des tribunaux ont finalement annulé l'interdiction de l'amiante moins de deux ans après qu'elle a été prononcé par l'agence.
} 
la preuve nécessaire pour le justifier, avec ses propres informations et données, puisque les industriels ne sont pas tenus de faire de tests particuliers pour la commercialisation.

L'OTS mit alors rapidement en œuvre l'approche structure-activité, recours presque unique dans cette situation où elle doit évaluer des produits sans données spécifiques. Charles Auer, un chimiste de formation, prit la responsabilité des activités structure-activité. En 1977, il s'adjoint les efforts de Joseph Seifter, figure de la pharmacologie, utilisateur expérimenté des méthodes quantitatives de conception de molécules au sein de l'industrie pharmaceutique. Seifter suggère de créer une « équipe structure activité » (structure-activity team), dédiée à cet exercice, dont Auer aura la responsabilité pendant plusieurs années. D’autres spécialistes de haut niveau de la prévision de la toxicité ou cancérogénicité des médicaments rejoignent l'EPA, en provenance de l'université ou de l'industrie pharmaceutique, en vue du travail de cette équipe. Elle est établie en 1979 au sein du bureau des substances chimiques, pour préparer l'arrivée des dossiers d'enregistrement des substances nouvelles (dits premanufacture notifications, ou PMN). Son utilité va rapidement être confirmée face à l'afflux de dossiers à traiter.

\section{La prédiction au cour de l'expertise sur les substances nouvelles}

Dès 1979 , les conditions de mise en œuvre du programme s'avèrent difficile. D’une part, les notifications de nouvelles substances reçues par l'agence ne contiennent que peu de données, et aucun résultat d'expérimentations ou de tests de toxicité ou d'écotoxicité (toxicité d'une substance pour les milieux environnementaux et les espèces qui y évoluent) qui auraient été conduits de manière ad hoc. D'autre part, et surtout, les dossiers se mettent à affluer en bien trop grand nombre pour que l'agence espère les expertiser dans le détail. Cet afflux de dossier est lié à la stratégie qui émerge dans l'industrie chimique par rapport à ce programme « nouvelles substances ». Les entreprises chimiques réalisent tôt que, la réglementation ne 
requérant de ne fournir qu'un minimum de données à l'EPA, la procédure de PMN peut être utilisée pour faire enregistrer par l'agence les molécules en développement, même celles dont les propriétés et usages commerciaux ne sont pas encore bien définis. Dès 1981, l'industrie se met donc à déposer des dossiers pour les substances en cours de développement. Elle fait, en quelque sorte, enregistrer son «pipeline» de recherche et développement, pour minimiser le travail d'enregistrement futur de ces substances. L'agence se retrouve ainsi à ordonner un marché de substances se comptant par milliers.

Ainsi, huit notifications sont soumises par des industriels à l'EPA en 1979, mais leur nombre augmente rapidement : 323 arrivent à l'OTS en 1980, 1379 en 1983, et jusqu'à 2993 dossiers en 1988 (Zeeman et al. 1993). Le flux de dossiers se stabilisera dans les années 1990 au niveau de 1600 notifications par an ${ }^{14}$. Dans la mesure où l'agence doit rendre un avis dans les 90 jours, cela signifie que le bureau doit chaque semaine faire un premier screening de plusieurs dizaines de substance, en l'espace de quelques jours seulement. Un ancien cadre du bureau des substances chimiques rappelle ainsi :

Quand je donne des présentations, je dis toujours la chose suivante : «imaginez que vous êtes évaluateur dans notre bureau. Vous arrivez au bureau le lundi et, cette semaine-là, ce sont cinquante produits chimiques qui doivent être évalués. Et il faut obtenir une réponse pour chacun d'entre eux ». En plus, tous ces produits sont différents. Mais il fallait prendre une décision, pour chacun, avant la fin de la semaine ou de la suivante. Au bureau, ces situations sont tout ce qu'il y a de plus courant's.

Cette situation impose au bureau d'utiliser l'expertise disponible parmi ses agents scientifiques, à partir des types de toxicité les plus fréquemment observées pour chaque forme moléculaire, et ce sans passer par des modèles permettant de vérifier statistiquement ces

\footnotetext{
${ }^{14}$ En septembre 2010, le bureau a reçu 50449 notifications. Globalement, la procédure de PMN a fonctionné de manière assez régulière depuis sa mise en place, avec environ 1600 notifications par an pendant ses 32 ans d'activité.

${ }^{15}$ Entretien avec un ancien toxicologue du bureau des substances chimiques, mai 2015.
} 
associations, dont le développement et la validation prennent plusieurs années. Il s'agit alors d'employer une analyse structure-activité de nature qualitative, où un toxicologue ou chimiste fait une analogie entre la structure d'une molécule nouvelle, et la structure d'une molécule dont il connait la toxicité, pour avoir mené des expérimentations ou considéré les résultats publiés d'expérimentations. Auer, à la tête de cette équipe de 1979 à 1986, se souvient qu'en son sein, « il y avait des gens capables de dire : bon sang, cette substance ressemble beaucoup à ce cas qu'on a étudié il y a un an ». Seifter, notamment, était un des experts de ce mode de raisonnement :

Il était l'un des premiers utilisateurs concrets des concepts qui visaient à former des catégories de substances chimiques et à chercher des valeurs/des gammes de toxicité qui traverseraient ces catégories. Il était sensible à la manière dont la toxicité pouvait évoluer au sein d'une catégorie, et ensuite capable de chercher les mécanismes qui sous-tendaient ces évolutions. [...] Vous pouviez lui montrer une structure moléculaire et il pouvait vous dire quels types d'effets cette substance était susceptible d'avoir sur la santé humaine.to

Le travail d'évaluation des relations structure-activité se formalise au cours du temps.

Nous avons organisé une première réunion. On avait à notre disposition la structure moléculaire de la substance, et peu ou pas d'informations disponible dessus (études animales, etc.). On a alors commencé un tour de table. En fonction de leur domaine d'expertise, on demandait à chacun ce qu'il pouvait offrir sur une substance particulière. Au fil du temps, cette pratique s'est régularisée et formalisée. On a pris l'habitude de décomposer l'examen des molécules, de les aborder avec des SAR [structure-activity relationships], puis de procéder à des restitutions communes."

Pendant les réunions, un chimiste était responsable de résumer le profil de la substance. Les propriétés physico-chimiques étaient ensuite examinées, puis ses effets sur l'environnement, ses effets sur la santé avant de finir par l'analyse de l'écotoxicité de la substance. Les

\footnotetext{
${ }^{16}$ Entretien avec Charles Auer, conduit par Jody Roberts, avril 2010.
}

${ }^{17}$ Ibid. 
inférences tirées des connaissances sur les propriétés des structures moléculaires comparables représentant alors une grande partie du travail des évaluateurs.

Les gens essayaient, dans l'idéal, de faire en sorte que les compétences des uns et des autres se complètent. Certains s'occupaient de la partie chimie, d'autres du métabolisme, mais ils parvenaient à se comprendre les uns les autres. Sur la base des trouvailles et des analyses de leurs collègues, ils parvenaient alors à comprendre la probabilité qu'un effet particulier survienne".

L'équipe passe rapidement de six à douze personnes, à mesure que les notifications commencent à affluer. Elle regroupe alors des chimistes, des chimistes environnementaux, des toxicologues et des médecins. Elle organise son travail autour de « réunions structureactivité » (structure-activity meetings), une délibération collective d'experts, qui deviennent rapidement une étape fondamentale du processus d'examen des notifications soumises par les entreprises. Elles se déroulent entre le $9^{\mathrm{inn}}$ et le $13^{\mathrm{im}}$ jour de la procédure d'examen standard, et examinent environ deux-tiers des dossiers reçus. Un calendrier «peu chargé » pour ces réunions comprend généralement cinq ou six substances, tandis que certaines réunions plus chargées comprennent jusqu'à vingt ou trente. Compte tenu de cette importante charge de travail concentrée sur quelques jours seulement, l'équipe mobilise souvent des prestataires extérieurs comme la Syracuse Research Corporation dont plusieurs employés ont ensuite rejoint les rangs de l'EPA.

Le résultat de ces réunions est l'identification des substances qui, comparativement à d'autres, méritent un examen plus poussé parce qu'elles tombent potentiellement sous la coupe du critère instauré par le TSCA : elles pourraient comporter un «risque non raisonnable ». La

\footnotetext{
${ }^{18}$ Les analyses structure-activité n'étaient cependant pas aussi efficaces pour tous les types de toxicité et pour tous les «endpoints » ou paramètres de toxicité. Elles sont surtout utiles pour les effets environnementaux et les problèmes d'écotoxicologie, et ce pour une raison simple : ce sont pour ces effets que des résultats expérimentaux à peu près standardisés et comparables, sur une diversité de substances, sont disponibles. Les tests écotoxicologiques sont menés sur des espèces de poisson se reproduisant rapidement, à la durée de vie courte, et au cout faible.
} 
structure-activity team classe les substances nouvellement examinées, passe ensuite à un autre groupe qui, au cours d'un «focus meeting » organisé entre le $15^{\circ}$ et $20^{\circ}$ jour de la procédure, conduit une évaluation du risque plus complète. À l'issue de celle-ci, il est décidé si un produit peut être fabriqué, doit être évalué plus en détail ou soumis à des mesures de contrôle.

\section{Construction mutuelle d'une alternative aux tests toxicologiques}

Les apparentes limitations de l'approche structure-activité poussent à interroger la manière dont industriels, scientifiques et fonctionnaires en arrivent à s'accorder sur son utilisation. Rien ne laisser présager du fait que l'approche SAR serait centrale. C'est au prix d'ajustements progressifs que ces acteurs construisent cette approche comme une alternative aux essais toxicologiques in vivo ou in vitro. Au départ, cette approche n'est pas consensuelle, ni au sein de l'EPA ni à l'extérieur. Au sein du bureau des substances chimiques, les personnes qui mettent en œuvre et défendent le raisonnement structure-activité, et qui s'engageront bientôt dans le développement de modèles structure-activité statistiques et validés, font face à un certain scepticisme des autres agents du bureau. En l'absence d'alternative, et sans veto explicite de leur direction, l'approche continue d'être appliquée.

A l'extérieur de l'agence, des réactions critiques sont apparues bien vite, par exemple au sein du Governmental Accounting Office ou de l'Office of Technology Assessment du Congrès, mais aussi parmi les chimistes de l'industrie. Les uns et les autres soulignent la fiabilité toute relative de ce genre de calculs, et le fait qu'il parait hasardeux de fonder des décisions réglementaires sur ces estimations non validées (Anonyme, 1984 ; EPA 1984 ; GAO, 1984; OTA, 1983). Corwin Hansch, interviewé dans une revue de l'American Chemical Society, juge négativement la pratique de l'EPA. D'une part, l'agence a l'ambition d'utiliser la méthode pour juger de multiples formes de toxicité à la fois, alors que la méthode est plus adaptée pour évaluer un «paramètre de toxicité » (endpoint) précis, sur lequel on dispose de 
données expérimentales et d'une bonne connaissance biologique théorique. L'outil, selon lui, est plus utile s'il est ciblé sur la vérification d'hypothèses précises. Par ailleurs, le manque de données physico-chimiques pour décrire les molécules ou de données expérimentales sur leurs effets toxicologiques hypothèque largement son utilité comme outil de décision réglementaire et commerciale. Au total, il ne pense pas «qu'on puisse encore baser de décisions réglementaires sur les SAR. En d'autres termes, cette approche n'est pas prête pour accepter ou refuser la mise sur le marché d'une quelconque substance » (Anonyme, 1984).

Ces critiques vont renforcer l'usage des relations structure-activité comme mode de raisonnement comparateur entre substances, par opposition à une modélisation statistique permettant de prédire avec précision les effets d'une substance en particulier. Adrien Albert, professeur à la Australian National University, spécialiste des relations structure-activité, expert invité aux structure-activity meetings en 1982, engage une évaluation du travail de l'EPA sur les relations structure-activité à la demande de l'agence. Il note que l'usage de ces associations, à l'EPA, est de nature qualitative, et qu'elle doit effectivement se limiter à ce travail de comparaison et de classification des substances, à partir de l'expérience accumulée des évaluateurs, sans s'aventurer dans l'application de modèles statistiques, à la crédibilité toujours limitée (EPA, 1984).

Les modèles statistiques sont encore considérés comme peu applicables à la décision publique sur des substances industrielles, dont le type et la toxicité ne sont pas forcément encore couverts par des modèles statistiques validés. À l'inverse, la pratique de catégorisation de substances en fonction d'indices de toxicité se trouve, elle, rapidement validée politiquement et pratiquement. Un ajustement mutuel de l'agence et de l'industrie se fait autour de l'idée selon laquelle le but de l'intervention sur ce marché étendu des nouvelles substances chimiques est de repérer les quelques substances potentiellement problématiques, et d'atteindre un niveau d'information uniforme suffisant pour éviter de fabriquer des substances 
controversées, telles les dioxines, formaldéhyde, benzène et autres phtalates, que le programme des «existing substances » a tant de mal à gérer au même moment. La visée commune de l'agence et des entreprises renvoie alors à l'un des objectifs de départ du TSCA : mettre fin un point final à la parade de «crises chimiques ${{ }^{19}}$ en mettant un place une procédure d'autorisation de mise sur le marché qui permette de repérer ces substances hautement toxiques (Davies et Davies 1970), celles qui suscitent la controverse dans les médias et alimentent ce que les fonctionnaires de l'agence appelaient alors le syndrome du

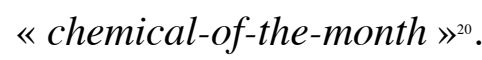

Cet ajustement mutuel et progressif se fait par trois voies simultanément. La première est celle des conférences scientifiques et multiples workshops réunissant scientifiques des agences et scientifiques des industries. Le département de recherche et développement de l'agence organisa de tels workshops tout au long des années 1970 et 1980, dans lesquelles se retrouvaient les chercheurs de ce département de l'agence, les chercheurs du bureau des substances toxiques, leurs supérieurs, ainsi que des chercheurs et chargés d'affaires réglementaires des entreprises chimiques. C'est surtout au sein des «Gordon Research Conferences »- une série de conférences dédiée à l'exploration collective des innovations dans le domaine de la chimie, qui débuta en 1931 et fut institutionnalisée grâce à l'appui de l'American Association for the Advancement of Science - que les chercheurs gouvernementaux et industriels pouvaient se croiser, débattre des applications des méthodes SAR et QSAR par l'EPA, et des meilleures stratégies pour tester de nouvelles familles de molécules. Les Gordon Research Conferences sur le QSAR débutèrent en 1975, et ont lieu tous les deux ans depuis, avec comme principe assumé de permettre le dialogue sur les

\footnotetext{
${ }^{19}$ Entretien avec J. Clarence Davies, conduit par Jody Roberts et Kavita Hardy, octobre 2009.

${ }^{20}$ Le terme renvoie à la succession d'affaires médiatiques autour de substances chimiques cancérogènes, qui mobilisait toute l'activité de l'agence pendant plusieurs semaines, avant de cesser, pour reprendre sur une nouvelle molécule quelques semaines plus tard. Au début des années 1980, le benzène, le formaldéhyde... ont chacune suscité ce genre d'épisodes.
} 
nouveaux fronts de recherche, entre chercheurs universitaires, d'agences gouvernementales et de l'industrie (Daemmrich et al. 2006). C'est en leur sein que, de leurs aveux, nombre de spécialistes de l'EPA ou de l'industrie, gagnèrent de l'expérience sur les applications de la méthode, les types de toxicité ou d'écotoxicité et les familles de substances sur lesquels elle était pertinente, et le type de déduction que les fonctionnaires de l'EPA étaient susceptibles de faire de ces données sur les substances nouvelles.

La deuxième voie est l'expérience des négociations directes engagées entre l'OTS et des entreprises individuelles pour la commercialisation de substances spécifiques. Au début de la mise en œuvre du TSCA, l'OTS est dans l'incertitude concernant sa capacité juridique à refuser la commercialisation d'un produit. Elle s'engage dans l'utilisation des méthodes structure-activité et des jugements par analogie sans savoir si ceux-ci seront acceptés par les industriels, et seront suffisamment crédibles pour résister à l'épreuve éventuelle des tribunaux en cas de plainte d'une entreprise requérante. Le bureau réalisa rapidement, au cours des années 1980, que les jugements structure-activité fonctionnaient comme une menace crédible. Si la loi n'impose pas d'obligations de test aux entreprises, les jugements d'analogie entre substances permettent précisément à l'OTS de formuler des hypothèses crédibles sur la toxicité des substances qui lui sont soumises, et de demander, dans un deuxième temps, des tests complémentaires. D'après le premier responsable du programme «substances nouvelles », l'approche structure-activité permet ainsi d'entreprendre une négociation délicate, dans laquelle l'industriel est menacé de poursuites judiciaires s'il refuse de fournir les informations demandées. Pour l'EPA, il s'agit d'une méthode risquée, parce que l'agence doit pouvoir justifier devant la Cour les raisons de son blocage, mais les risques sont également élevés pour les industriels visés :

Elles savent que si elles poussent l'EPA à en arriver là, l'agence risque de le leur faire payer pour toutes leurs futures PMN. Contrairement à d'autres programmes, on voit les mêmes entreprises encore et encore, et ces 
entreprises veulent bien sûr avoir une bonne relation avec l'agence. Même si elles l'emportent [en justice] contre l'EPA, leur nouvelle substance est condamnée. Il n’y a pas de place sur le marché pour une substance qui a des problèmes avec l'agence. C'est aussi simple que ça ${ }^{21}$.

Au début des années 1980, l'agence reçoit par exemple un dossier d'enregistrement qui concerne une substance de la famille des phtalates — des molécules très utilisées pour leurs propriétés plastifiantes, notamment dans la production du PVC. L'équipe du bureau des substances chimiques en sait alors suffisamment sur les relations structure-activité pour déterminer qu'il s'agit d'un analogue du DEHP, un des phtalates les plus répandus et dont le potentiel cancérogène est alors sous le feu des projecteurs. Dans ces situations, l'agence se trouve en position de force :

D'après la section 5 du TSCA, si une entreprise refuse de coopérer, ce que l'EPA peut en théorie proposer c'est de prendre une décision qui dit « jusqu'à ce que vous nous fournissiez telles données, votre utilisation est interdite ou bien vous ne pouvez pas produire du tout $»^{22}$.

Dans ce cas, l'entreprise avait jugé les essais demandés trop coûteux, et retiré sa demande d'enregistrement $^{23}$. La majeure partie du temps, les dépenses de $R \& D$ des entreprises en amont de la demande d'enregistrement étant limitées (puisqu'aucun essai n'était obligatoire dans la loi), les dépenses supplémentaires demandées par l'EPA paraissent cependant acceptables. Les industriels acceptent ainsi généralement de réaliser les expérimentations supplémentaires requises par l'agence, si un soupçon émerge qu'une substance pourrait être toxique. C'est finalement dans les cas où le cout des tests demandés excèdent la promesse commerciale de la molécule candidate à la commercialisation que les entreprises préfèrent abandonner leur demande et examiner d'autres pistes chimiques.

\footnotetext{
Entretien avec un ancien responsable du programme « substances nouvelles » de l'EPA, mars 2012.

Ibid.

${ }^{23}$ Ibid.: «Des années après mon départ de l'EPA, j'ai déjeuné avec un des avocats qui travaillait pour cette entreprise. A un moment il me dit 'vous savez, c'est vraiment dommage que vous ayez tué cette molécule', et j'ai répondu 'on ne l'a pas tuée, on a juste demandé des essais sur sa toxicité'. Selon lui, 'bien sûr, mais il y en avait pour 2 millions de dollars d'essais simplement pour une molécule. Jamais on n'aurait fait ça !' »
} 
La troisième et dernière voie est celle de la collaboration institutionnelle entre l'OTS et des instances collectives de l'industrie, notamment la Chemical Manufacturers Association $(\mathrm{CMA})^{24}$. C'est sous l'égide de ce syndicat que les entreprises qui fabriquent des produits similaires ou de la même famille se regroupent, au cours des années 1980, dans des panels dédiés. Ces panels engagent alors des discussions avec des représentants de l'EPA, généralement avec les fonctionnaires de l'OTS examinant les dossiers de demande de commercialisation. Ce qui sera baptisé dans les documents de l'agence le «EPA-Industry Trade Dialogue » donne lieu à l'élaboration d'accords formels sur des programmes de tests, que les industriels déploient, pour ensuite transmettre les données en résultant à l'agence. Les producteurs d'acrylate et méthacrylate composèrent ainsi un panel, qui dialogua avec l'agence pendant deux longues années (1987-1989), pour aboutir à un programme de production de données sur une vaste catégorie de substances - données grâce auxquelles l'agence pouvait engager des jugements comparatifs entre les substances de cette famille (EPA 1990).

Malgré les critiques initiales dont la méthode a été l'objet, l'agence est progressivement confortée dans sa pratique des relations structure-activité comme méthode de consultation du catalogue mental de structures moléculaires et d'effets toxiques des toxicologies et chimistes de l'agence, pour classer ces substances en familles de produits plus ou moins toxiques, grâce aux ajustements avec les industriels que permet d'opérer ce tissu serré de relations qui, dans leur variété (relations agence/industrie médiée par leurs scientifiques respectifs, relations directes entre membres de l'OTS et des firmes individuelles, ou enfin relations institutionnelles entre dirigeants de l'OTS et syndicats industriels), permettent un apprentissage stratégique mutuel. Ces expériences confortent la procédure instaurée à l'agence : le raisonnement structure-activité ne définit pas la décision finale de l'agence de

${ }^{24}$ Le syndicat de l'industrie chimique américaine s'appelait Manufacturing Chemists Association jusqu'en 1978 et porte le nom d'American Chemistry Council depuis 2000. 
bloquer une substance ou de la laisser entrer en commercialisation. Il sert à profiler la substance, et à l'orienter vers une des voies de l'arbre décisionnel : autoriser la substance, ou demander plus de données de l'industriel concerné, pour pouvoir mener une évaluation plus complète.

Crédibiliser et standardiser le raisonnement structure-activité : les projets QSAR et ECOSAR renvoient le raisonnement à l'industrie

Cet ajustement agence-industrie est perceptible dans deux autres développements ultérieurs. Le premier est l'investissement dans la validation de modèles de relations structure-activité quantitatifs. Comme les critiques de l'année 1984 l'ont montré, le scepticisme reste alors grand sur l'idée de pouvoir prédire la toxicité à partir de relations statistiques entre descripteurs moléculaires et toxicités observée expérimentalement, sur de grands ensembles de molécules, en tout premier lieu car il n'existe quasiment pas de programmes de test standardisé sur de tels grands ensembles. C'est pourtant dans un tel programme que 1'EPA s'engage progressivement, pour produire et appliquer petit à petit, dans la décision réglementaire, des modèles validés.

En dehors du travail d'évaluation, les bureaucrates des relations structure-activité déploient en effet une grande énergie pour le développement d'outils et de modèles quantitatifs de relation structure-activité. Bob Lipnick, un écotoxicologue de l'agence, commence à utiliser ces approches dès 1981. Avec le soutien du chef de la Environmental Effects Branch, il se lance alors dans l'analyse en série de 55 alcools avec un modèle QSAR connu pour la narcose ${ }^{25}$ publié dans la littérature. Ce modèle lui permet de déterminer la valeur pour laquelle un niveau de narcose apparaît en fonction de certaines structures moléculaires. En comparant les

\footnotetext{
${ }^{25}$ Donc pour sa capacité à endormir en provoquant artificiellement le sommeil.
} 
nouveaux alcools avec ces structures moléculaires, il devient alors possible de dire si ces alcools peuvent provoquer la narcose.

Les recherches conduites par Lipnick et beaucoup d'autres ont largement alimenté les réunions structure-activité. Lipnick participait à ces réunions avec les résultats de ses estimations. Du côté de l'Office of Research and Development (ORD), un vaste programme de test de 617 substances sur une espèce de poisson à développement rapide (le « fathead minnow », donnant ce qui sera appelé à l'agence la «fathead minnow database ») déboucha dans le cours des années 1980s sur la production d'une cinquantaine de modèles statistiques considérés comme suffisamment robustes pour être utilisés au cours des réunions structureactivité. Les résultats du programme d'essais furent présentés dans une série de publications écrites par les spécialistes des relations structure-activité de l'OTS et préfacées par Gil Veith, un de leurs principaux promoteurs. Dans la première publication de cette série, en 1988, il note que les :

«Les scientifiques dans des conditions normales sont chanceux, ils ont des données. Mais les scientifiques avec des responsabilités réglementaires, eux n'en ont pas. Ces scientifiques réglementaires ont donc cherché à répondre aux obligations réglementaires faites à l'EPA en adaptant dans ce contexte des approches utilisées dans la conception de médicaments et en chimie théorique pour prédire le comportement et la toxicologie de molécules sur la base de leur structure plutôt qu'avec l'aide d'un grand nombre de données. L'ORD et le Bureau des substances chimiques ont collaboré pendant plus de dix ans pour développer des relations structure-activité capables d'estimer le potentiel de bioaccumulation, la persistance et la toxicité des molécules dans l'environnement. » (Veith 1996)

Le partenariat entre OTS et ORD fonctionnait donc de la manière suivante : le bureau responsable du programme substances chimiques fournissait des fonds à l'ORD pour qu'il développe ses bases, et l'ORD partageait en retour ses résultats avec le bureau de manière à ce que ses membres puissent identifier de nouvelles corrélations structure-activité et mettent en place des programmes informatiques capables de prédire, sur cette base, les valeurs de 
toxicité. Entre 1981 et 1983, au début des études «fathead minnow », treize QSAR furent créées. Dix ans plus tard, 49 QSAR différentes étaient disponibles pour estimer la toxicité aquatique et le potentiel de bioconcentration de trente classes ou sous-classes de produits chimiques industriels.

L'autre développement concerne la mise au point d'un outil logiciel pour soutenir le travail d'analyse des relations structure-activité, et le mettre en œuvre sur de plus grandes masses de données. Les agents du bureau des substances chimiques ont joué un rôle majeur dans le développement de cet outil, au départ simple «manuel de référence QSAR » regroupant les informations relatives aux treize premiers QSAR développés par le bureau. Le second manuel fut publié en 1988 et fut, cette fois, rendu public. Il incluait 49 QSAR pour 30 classes chimiques. L'espoir du bureau, à ce stade, était de faire circuler cet outil. Comme le rappelle le chef de la Environmental Effects Branch de l'époque ${ }^{26}, 800$ copies furent distribuées. Le document n'était pas excessivement cher, de manière à permettre une diffusion plus facile. Une version révisée du manuel QSAR fut produite en 1993, et celle-là constitua un tournant. Jusque-là, il ne s'agissait que d'une version papier. En 1994, une version informatique du manuel, ECOSAR, fut rendue publique. Cette version contenait 42 classes chimiques et 120 QSAR. L'idée de numériser le manuel venait de Dick Clements, un des chimistes du bureau, au cœur de cette équipe de «QSAR folks », et amateur de la bioinformatique naissante.

ECOSAR était vu comme une réponse au problème de la diffusion du manuel dans l'industrie. Le manuel papier comptait plusieurs centaines de pages, et n'avait pas amené de changement notable dans la composition des dossiers industriels : on y trouvait peu d'analyses SAR ou QSAR. Le logiciel devait permettre un recours facile au screening des molécules sous l'angle structure-activité, pour produire des données non couteuses, avant la notification. Si

${ }^{26}$ Entretien avec l'ancien chef de l'Environmental Effects Branch, mai 2015. 
l'industrie n'a aucune obligation de fournir des données, des outils permettant de réaliser à l'avance, en interne, le type d'évaluation que l'agence allait appliquer à ces produits ensuite, étaient eux tout à fait susceptibles d'être adoptés. ECOSAR est devenu un des logiciels les plus usités à travers le monde pour calculer les descripteurs physico-chimiques des substances, trouver des substances analogues, ou faire tourner certains modèles QSAR (Boullier et Demortain 2017). Sa diffusion a fait des SAR et des QSAR une forme d'analyse conjointe industrie-autorité, dans laquelle les sociétés produisent une première analyse, que l'autorité évalue, audite et corrige. Aujourd'hui encore, le logiciel est activement distribué dans le cadre du programme « futurs durables » de l'EPA dont un des objectifs est de faire en sorte que ces outils soient adoptés par les entreprises.

\section{Science et marché dans la construction de l'expertise scientifique des produits}

L'histoire de l'utilisation de la méthode structure-activité à l'EPA montre que cette expertise s'est construite en même temps que les acteurs, administratifs comme industriels, découvraient l'ampleur du marché et la diversité des substances qui étaient susceptibles d'être commercialisées. Elle reflète la compréhension naissante des stratégies de R\&D des acteurs industriels, et la connaissance globale, elle aussi progressivement constituée, de la variété et de la nature des objets à contrôler. Elle est ajustée, enfin, au niveau d'intervention publique sur l'industrie défini par la loi, et réglé dans le cours des rapports continus entre l'agence et les entreprises chimiques dans la mise en œuvre du programme.

Quatre moments illustrent ce modelage de l'expertise scientifique des produits sur une analyse du marché en constitution, et dans le réseau des relations entre les acteurs qui construisent ce marché - ici les producteurs des biens, l'agence qui réglemente leur commercialisation, et les scientifiques qui évaluent les produits. Le premier est le repérage de 
la méthode des relations structure-activité dans les pratiques de l'industrie pharmaceutique en réponse au travail politique de l'industrie pour réduire ses obligations de test dans le cadre du TSCA, et l'apprentissage de cette méthode par l'EPA, grâce au recrutement d'experts venant de cette industrie. Le second est l'institutionnalisation de l'équipe et des réunions structureactivité en réaction à la multiplication des demandes de mise sur le marché dont l'agence fait l'expérience concrète dès le début des années 1980. Le troisième est l'ajustement du niveau de preuve recherché à travers les relations structure-activité, suivant un accord implicite sur l'objectif de l'intervention publique sur l'industrie - lisser l'innovation, signaler les produits les plus mauvais, éviter les «chemical crises » - et l'évaluation de la qualité des prédictions permises par les modèles statistiques, jugés immatures. Le quatrième et dernier moment est celui de la stabilisation des bases de données et des outils de calcul des relations structureactivité, notamment le manuel ECOSAR, comme moyen de rediffuser dans l'industrie un raisonnement préparatoire à l'autorisation.

Si l'utilisation du raisonnement structure-activité à l'EPA pour expertiser les produits chimiques n'est évidemment qu'un cas particulier, on peut néanmoins faire l'hypothèse que cette succession d'ajustements mutuels entre l'agence et l'industrie chimique dessine un processus historique caractéristique de la manière dont se construit l'expertise scientifique pour l'action publique, à visée de réglementation des produits et des risques. L'analyse historique faite ci-dessus montre que l'expertise scientifique est bien le reflet des anticipations marchandes formées de manière interactive entre les acteurs industriels, scientifiques et administratifs participant à la qualification de ces produits aux qualités incertaines, au cours du temps - un réseau d'anticipations. On peut approcher l'expertise scientifique réglementaire sous l'angle du «calcul » économique, et de la constitution des marchés par le calcul, comme la sociologie économique nous y invite. Si l'expertise examinée ici bien pour ambition de simuler la réalité de manière aider la prise de décision (Angeletti 2011, Aykut 2019), les outils 
qu'elle mobilise ne cherchent pas directement à établir des scénarios sur le futur comme cela a été largement étudié dans le cas du changement climatique (Dahan, 2007) ou de la prévision économique (Angeletti, 2012 ; Canu et Ducourant, 2018). On peut considérer que les repères cognitifs que constituent les anticipations marchandes analysées ici permettent aux acteurs d'envisager la réalisation de leurs objectifs et de leur intérêt, et donc guident leurs choix du moment (Beckert 2012, 2016). En l'occurrence, on observe trois choses.

D’une part, le recours à des analyses structure-activité d'abord qualitatives, par consultation d'experts humains (plutôt que de systèmes-experts qui calculeraient la toxicité à partir de modèles statistiques), ne s'impose pas a priori sous l'influence de scientifiques qui en défendraient la validité. Il fait suite à une anticipation de l'industrie chimique du risque que représente le TSCA du point de vue de la commercialisation de nouveaux produits. Cette anticipation conduit à un travail de lobbying visant la réduction à la baisse des obligations de test préalable de ses produits. Il fait également suite à l'anticipation de l'agence américaine, en réaction au comportement déployé par l'industrie, des difficultés à évaluer les produits sans données. Il est confirmé après des évaluations émanant de différents organismes, mais aussi de chimistes experts, internes ou externes à l'agence, de l'applicabilité et de la crédibilité des modèles statistiques dans un cadre de décision juridique. Il est encore confirmé par le fait que les demandes de données aux industriels, justifiées par des jugements de type structure-activité, étaient acceptées par les entreprises. L'évaluation de produits incertains est donc indissociable de l'évaluation, au fil de l'eau, par l'agence, de sa capacité à mettre en œuvre le programme, et à faire passer ses décisions de restriction ou de noncommercialisation auprès des requérants et, le cas échéant, des tribunaux.

Deuxièmement, différents acteurs produisent ces anticipations concernant les nouveaux produits, et s'influencent les uns les autres à travers elles. Trois acteurs en produisent. Les fonctionnaires de l'agence, qui estiment que le bénéfice d'appliquer une méthode d'analyse 
des SAR et de catégorisation des produits chimiques pourtant très personnalisée, dépendante de l'expertise incorporée des membres, dépasse le risque de ne pas réussir à traiter la masse des dossiers affluant. L'agence, qui préfère ébaucher des décisions sur la base d'une méthodologie innovante, même expérimentale, et risquer une mise en cause au tribunal pour une décision particulière, que d'être accusée au Congrès et dans les médias, d'avoir failli dans la mise en œuvre du programme nouvelles substances dans son ensemble. L'industrie ellemême, dont le calcul initial d'envoyer à l'agence des dossiers pour la plus grande partie de ces molécules en développement, a été déterminant dans le développement et l'application de la méthode SAR. Les acteurs scientifiques, collectivement, qu'ils travaillent pour l'agence, à l'université ou auprès des industriels, produisent et mettent en circulation de telles anticipations. On a notamment relevé l'intervention de Corwin Hansch, chimiste computationnel éminent, évaluant l'intérêt et la faisabilité d'appliquer des modèles statistiques dans le cadre de la réglementation, mais aussi celle de Adrian Albert, au service de l'EPA pendant quelques mois dans les années 1980. Ces anticipations et ces calculs politiques se répondent. Les acteurs s'ajustent les uns aux autres en fonction de ceux-ci, de telle manière qu'on peut parler $\mathrm{d}^{\prime}$ « anticipations croisées ». Ces interactions entre acteurs ainsi que les anticipations croisées qu'ils produisent sur la réglementation des produits et les procédés d'évaluation, forment le tissu relationnel dans lequel se constitue le marché, de manière à la fois concrète (les décisions de mise sur le marché des produits) et symbolique.

Troisièmement, on observe une co-construction de l'intervention publique par l'administration et l'industrie. En écho aux discussions actuelles sur l'étendue de la «capture» des autorités réglementaires par les industries (Carpenter et Moss 2014), il apparait ici que l'opposition autorités/entreprises n'est pas aussi centrale que le processus de construction d'un raisonnement, et d'équipements, capables de qualifier des objets, leurs risques, et d'organiser leur commercialisation. Ce raisonnement n'est ni privé ni public, mais 
construit dans l'interaction entre des acteurs dont les logiques relèvent de l'une ou l'autre sphère d'activité, et qui anticipent les actions des uns et des autres dans leurs choix.

La trajectoire des relations structure-activité à l'EPA est révélatrice de l'intense travail d'ajustement nécessaire au maintien d'un système réglementaire aux objectifs apparemment contradictoires de protection de la santé et de l'environnement d'une part, et de commercialisation de marchandises à risques d'autre part. L'origine même des SAR, empruntées à l'industrie pharmaceutique, a favorisé son acceptation comme forme de savoir capable de circuler aisément entre les acteurs impliqués dans l'évaluation des molécules nouvelles. Progressivement, le recours à cette approche moins onéreuse que les essais toxicologiques classiques permet aux administrations et aux entreprises de s'accorder sur un mode de connaissance des qualités des produits qui génère des données nouvelles tout en participant à l'arrivée sur le marché de plusieurs dizaines de milliers de nouvelles molécules. Tout le travail des tenants de l'approche SAR consiste ainsi à raisonner à l'échelle de l'alerte et à ne jamais aller trop loin dans le raisonnement toxicologique. Le maintien d'un tel positionnement au fil du temps facilite le travail d'articulation auquel les acteurs se livrent, entre protection de la santé et de l'environnement et développement du marché de la chimie. Le positionnement de l'approche SAR dans le champ scientifique n'est d'ailleurs pas étranger à son développement et son adoption : il ne s'agit ni de chimie académique fondamentale (même si les hypothèses viennent partiellement de là), ni d'une science réglementaire parfaitement standardisée (aujourd'hui encore, les critères de production des SAR sont assez lâches). Ces caractéristiques contribuent à en faire une méthode complémentaire, dans un assemblage d'informations constituant un faisceau de preuves - on parle de «weight of evidence » - tout juste suffisant pour produire une alerte et pour faire échanger industriels et régulateurs et organiser le marché. 


\section{Conclusion}

Cet article propose une analyse historique du recours à une forme d'expertise scientifique innovante à l'agence environnementale américaine, institution qui a inauguré l'utilisation de méthodes issues de la chimie computationnelle dans l'évaluation des produits chimiques. La question empirique posée dans l'article était de comprendre pourquoi l'expertise computationnelle s'impose, et pourquoi elle prend une forme donnée - en l'occurrence une forme qualitative, fondée sur l'interrogation de l'expertise accumulée par les toxicologues ou chimistes de l'agence, presque incorporée et tacite, et seulement progressivement formalisée à travers des modèles statistiques validés et un outil informatique. La question théorique associée était de savoir comment le contexte marchand influence la constitution et l'usage pratique d'une science pour l'évaluation et l'action publique ; question justifiée par le fait que la littérature sur les savoirs scientifiques dans l'action publique ont très largement ignoré cette dimension.

Le fait principal sur lequel s'est appuyée l'analyse est que l'agence environnementale américaine a, dans les termes d'un de ses fonctionnaires, recouru aux méthodes structureactivité par nécessité. Elle n'avait que cette méthode à sa disposition pour traiter de grands lots de dossiers contenant un minimum d'information spécifique sur chaque substance. Seule cette méthode lui permettait de construire des preuves scientifiques au niveau requis par la loi - donner une suspicion raisonnable de risque pour pouvoir requérir des industriels des données supplémentaires. Il est indéniable que la loi TSCA créait un faisceau de contraintes très étroites pour l'agence (responsabilité de la preuve, dans un temps très court, et sans données, des risques de substances multiples). Pour autant, cette nécessité n'a pas imposé à l'agence, de manière exogène, d'utiliser une science prédéfinie. La solution trouvée est le résultat d'un travail de recherche, et a pris forme au cours d'un processus de calcul croisé 
avec l'industrie. Elle s'est imposée en enquêtant sur ce que l'industrie elle-même faisait, en réagissant à sa stratégie d'inondation de l'agence avec des dossiers vides, et à mesure que les décisions étaient acceptées par l'industrie.

Les savoirs et les instruments produits et assemblés dans ce contexte forment ce qu'on pourrait appeler une expertise de marché. L'expertise scientifique mobilisée dans ce contexte réglementaire consiste en un travail de qualification qui vise à déterminer les modalités de commercialisation d'objets techniques. Alors que ces interactions sont le plus souvent analysées en termes d'influence ou de rapports de pouvoir, l'article montre que c'est au fil d'ajustements entre les acteurs impliqués dans la fabrique de cette expertise (fonctionnaires de l'agence, scientifiques, firmes chimiques) que la modélisation structure-activité est construite comme acceptable, crédible et finalement efficace. C'est en fonction de ces interactions de marché que se décident la forme concrète de cette connaissance (structure-activité qualitative plutôt que des modèles statistiques, introduits progressivement), et les développements à mener à bien (la création d'outils logiciels pour systématiser l'application de la méthode dans l'industrie).

Replacer l'expertise scientifique des produits et des risques dans le cœur des relations constitutives du marché permet de faire avancer à la fois la sociologie politique de l'expertise, et la sociologie économique de l'action administrative, ou de l'État plus généralement. Sur l'expertise elle-même, plus particulièrement sur la mise en place de « situations d'expertise » et l'émergence de la crédibilité d'un type de savoir pour l'action publique, on comprend ici que cette situation n'est pas qu'une intermédiation entre science et politique, une rencontre entre une offre universitaire de savoirs et le besoin d'une agence réglementaire de résoudre une situation problématique. L'expertise des produits et de leurs risques émerge dans une situation qui se construit à la confluence d'une compétence scientifique (la chimie computationnelle, drug design), d'une problématique administrative (mettre en ouvre le 
TSCA) et du marché qui émerge - la somme des produits à évaluer et l'incertitude qui les entoure.

Si le développement d'un produit prometteur à l'aide de méthodes computationnelles de drug design fondées sur les relations structures-activité est né dans l'industrie pharmaceutique et parmi ses chimistes, l'évaluation de produits sur base de relation structure-activité telle que l'EPA la met en pratique n'a pris forme que dans l'interaction entre l'agence, les industriels et des scientifiques consultés dans le cours de ce travail méthodologique, donc entre des acteurs publics, d'autres privés, d'autres qui ne sont ni l'un ni l'autre ou qui sont l'un et l'autre. Si l'on peut parler d'expertise de marché, c'est parce que ce mode de connaissance des produits est construit dans le cours de la formation d'un marché, de la définition de qualités d'une gamme de produits amenés à faire l'objet d'un échange, entre les acteurs dont dépend l'établissement de ces qualités et leur mise en circulation. 


\section{Références}

ANGELETTI T., 2011, « Faire la réalité ou s’y faire ? La modélisation et les déplacements de la politique économique au tournant des années $1970 »$, Politix, 95, p. 47-72.

ANGELETTI T., 2012, « La prévision économique et ses erreurs », Raisons politiques, 48, p. 85-101.

AYKUT S.C., 2019, Reassembling Energy Policy: Models, Forecasts, and Policy Change in Germany and France." Science \& Technology Studies Forthcoming.

ANONYME, 1984, "Structure activity relationships: Are they predictive of toxicity and potential regulatory tools? », Environmental Science and Technology ,18, 9, p. 285-286.

ANSAlONi M., SMith A., 2017, «Des marchés au service de l'État?», Gouvernement et action publique, 4, p. 9-28.

BARRY A., 2005, "Pharmaceutical Matters: The Invention of Informed Materials." Theory, Culture \& Society 22, 1, p.51-69.

Beckert J. 2012, Anticipations fictionnelles en économie. Perspectives, 8, p. 3-5.

BECKERT J., 2016, Imagined Futures. Harvard University Press.

BENOIT C., 2018, Le pouvoir de régulation transnational d'une agence nationale. Gouvernement et action publique, 1, p. 9-32.

Bensaude-Vincent B., Stengers I., 2001, Histoire de la chimie. La Découverte.

Borraz O., Demortain D., 2015, «Science Réglementaire » In Dictionnaire Critique de l'expertise. Santé, Travail, Environnement, edited by Emmanuel Henry, Claude Gilbert, Jean-Noel Jouzel, and Pascal Marichalar, 281-88. Presses de Sciences Po.

Boudia, S., Demortain D., 2014, «La Production d'un Instrument Générique de Gouvernement. Le 'Livre Rouge' de l'analyse Des Risques », Gouvernement \& Action Publique 3, 3, p. 33-53.

Boudia, S., Demortain D., 2015. "Evaluation Des Risques.” In Dictionnaire Critique de l'expertise. Santé, Travail, Environnement, edited by Emmanuel Henry, Claude Gilbert, Jean-Noel Jouzel, and Pascal Marichalar, 134-41. Presses de Sciences Po.

Boullier H., 2016a, «Évaluer des dossiers «vides »: L'expertise REACH face aux asymétries d'information ». Terrains \& travaux, 28, 1, p. 41-61.

Boullier H., 2016b, «Gouverner les molécules «sans données ». Généalogie de l'interdiction par l'autorisation dans REACH », Annales des Mines - Gérer et comprendre, 126(4), 61-70.

BOULliER H., 2016c, Autoriser pour interdire. La fabrique des savoirs sur les molécules et leurs risques dans le règlement européen REACH. Thèse pour le doctorat de sociologie, Université Paris-Est.

BOUllier H., 2019, Toxiques légaux, Paris, Éditions La Découverte.

Boullier H. Demortain D., 2017, «Économie de la prédiction : les outils logiciels pour QSAR et read-across », communication présentée à la journée d'étude d'étude Produire la prédiction: le travail de la modélisation pour l'évaluation des risques, Paris, 19 juin 2017. 
Callon M., Meadel C., Rabeharisoa V. 2000, «L'économie des qualités », Politix 13, 52, p. 211-239.

Callon M., Eymard-Duvernay F., Gadrey J., Karpik L., Musselin C., Paradeise C., 2002. La qualité. Sociologie du travail, 44, p. 255-287.

CAnu R., Ducourant, H., 2018, «Genèse des futurs économiques », Revue Française de Socio-Économie, 21, 2, p. 27-38.

CARPENTER D., 2010, Reputation and Power: Organizational Image and Pharmaceutical Regulation at the FDA. Princeton, NJ: Princeton University Press.

CARPenter D., Moss D., 2014, Preventing Regulatory Capture: Special Interest Influence and How to Limit It. Cambridge and London: Cambridge University Press.

Clements R., NABholz V., Johnson W., et al., 1993, "The Use and Application of QSARs in the Office of Toxic Substances for Ecological Hazard Assessment of New Chemicals", In: Landis W, Hughes $\mathrm{J}$, and Lewis M (eds) Environmental Toxicology and Risk Assessment. Philadelphia: American Society for Testing and Materials, pp. 56-64.

Creager A., 2018, Testing Toxics: Tools, Standards, and Corporate Data in the History of Chemicals Regulation. In: Pervasive Powers. Corporate Authority in the Shaping of Public Policy, Université Paris Dauphine, 16 June 2018.

Crum-Brown A.C., Fraser T.R., 1868, On the Connection between Chemical Constitution and Physiological Action; with special reference to the Physiological Action of the Salts of the Ammonium Bases derived from Strychnia, Brucia, Thebaia, Codeia, Morphia, and Nicotia. Journal of Anatomy and Physiology 2, 2, p. 224-242.

DAEMMrich A.A., GRAY N.R., SHAPER L., 2006, Reflections from the Frontiers, Explorations for the Future: Gordon Research Conferences, 1931-2006. Chemical Heritage Foundation: Philadelphia.

Demortain D., 2009, "Standards of Scientific Advice. Risk Analysis and the Formation of the European Food Safety Authority." In Scientific Advice to Policy Making: International Comparison, edited by J. Lentsch and P. Weingart, 141-59. Berlin: Barbara Budrich.

DEMORTAIN D., 2017, "Expertise, Regulatory Science and the Evaluation of Technology and Risk: Introduction to the Special Issue.” Minerva, 55, 3, p. 139-59.

Demortain D, 2017, Designs on Controversy. A Political History of Risk Governance as the US EPA, Mémoire inédit pour l'habilitation à diriger des recherches, Université Paris-Est.

Demortain, David. 2020. The Science of Bureaucracy. Risk Decision Making and the US Environmental Protection Agency. Cambridge ; London: MIT Press.

DAHAN A., 2007, «Les modèles du futur. Changement climatique et scénarios économiques : enjeux scientifiques et politiques », La Découverte, Paris.

DAviEs, C.J., 1970, The Politics of Pollution. Indianapolis: Bobbs-Merrill.

DUBUISSON-QUELLIER S., 2017, La capture comme levier de l'intervention publique sur l'économie: Le cas de la politique publique d'affichage environnemental en France. Revue française de sociologie ,58, 3p. 475.

EPA, 1981, State-of-the-art report on structure activity methods development. 600/3-81-29, April. Prepared by Environmental Research Laboratory, for the Office of Toxic Substances, United States Environmental Protection Agency. 
EPA, 1990, Accomplishments and Goals in Reducing Toxic Chemical Risk. FY 1989 Report to Congress on the Toxic Substances Control Act (TSCA), 21T-2001, Washington DC: Pesticides and Toxic Substances (TS-799), US Environmental Protection Agency.

EPA, 1994a, ECOSAR: Computer Program for Estimating the Ecotoxicity of Industrial Chemical Based on Structure Activity Relationships, Users Guide. 748-R-93-002. Washington D.C.: Office of Pollution Prevention and Toxics, US Environmental Protection Agency.

EPA, 1994b, Estimating Toxicity of Industrial Chemicals to Aquatic Organisms Using Structure Activity Relationships 2nd Edition. EPA-R-93-001. Washington D.C.: Office of Pollution Prevention and Toxics, US Environmental Protection Agency.

EYMARD-DUVERNAY François, GADREY Jean, et KARPIK Lucien, 2002, « La qualité », Sociologie du travail, 44, p. 255-287.

FAULKNER Alex, 2012, «Commensuration and Proliferation: Similarity and Divergence in Law's Shaping of Medical Technology », Law, Innovation and Technology, 4, 2 , p. 165 184.

FAULKNER Alex, 2017, "Special Treatment? Flexibilities in the Politics of Regenerative Medicine's Gatekeeping Regimes in the UK », Science as Culture, 28, 2, p. 149-173.

FRI, 1975, Structure activity correlation bibliography, by the Franklin Institute Research Laboratories, for the Office of Toxic Substances.

FRI, 1976. Analysis and trial application of correlation methodologies for predicting toxicity of organic chemicals", technical report by requested by the Office of Toxic Substances from the Franklin Institute Research Laboratories, EPA/560-1-76-006.

FRICKEL Scott et MOORE Kelly (dir.), 2006, The new political sociology of science: institutions, networks, and power, Madison, University of Wisconsin Press.

GAO, 1984, Assessment of New Chemical Regulation Under the Toxic Substances Control Act. GAO/RCED-84-84, 15 June. General Accounting Office, Washington, DC (USA). Office of the Comptroller General.

GENSCHEL Philipp et ZANGL Bernhard, 2011, «L'État et l'exercice de l'autorité politique Dénationalisation et administration », Revue française de sociologie, 52, 3, p. 509-535.

GRANJOU Céline, 2004, « Le travail des experts : analyse d'un dispositif d'évaluation des risques alimentaires », Sociologie du Travail, 46, 3, p. 329345.

HAURAY Boris, 2015, «Conflit d'intérêts », in Emmanuel Henry, Claude Gilbert, Pascal Marichalar, et Jean-Noel Jouzel (dir.), Dictionnaire critique de l'expertise. Santé, environnement, travail, Presses de Sciences Po, p. 7179.

HENRY Emmanuel, 2017, Ignorance scientifique et inaction publique: Les politiques de santé au travail, Presses de Sciences Po.

IRWIN Alan, ROTHSTEIN Henry, YEARLEY Steven, et MCCARTHY Elaine, 1997, « Regulatory science-Towards a sociological framework », Futures, 29, 1, p. 1731.

JASANOFF S., 1995, « Procedural choices in regulatory science », Technology in Society, 17, 3, p. 279293.

JASANOFF Sheila, 1987, "Contested Boundaries in Policy-Relevant Science », Social Studies of Science, 17, 2, p. 195230. 
_- - , 1990, The Fifth Branch: Science Advisers as Policymakers, Boston, MA, London, Harvard University Press.

JOLY Pierre Benoit, 2005, « La sociologie de l'expertise scientifique: les recherches françaises au milieu du gué », in Risques, crises et incertitudes : pour une analyse critique, MSH Alpes, p. 117174.

JOLY Pierre-Benoît, 2012, « La fabrique de l'expertise scientifique : contribution des STS », Hermès, La Revue, 64, 3, p. 2228.

- - - 2016, « Science réglementaire: une internationalisation divergente ?, Regulatory science: a divergent form of internationalisation? The evaluation of biotechnology in the United States and Europe », Revue Française de Sociologie, 57, 3, p. 443472.

JUDSON Richard, WAMBAUGH John, ISAACS Kristin, et THOMAS Russell, 2018, Computational and Molecular Approaches to Risk Assessment, .

KING Desmond et GALES Patrick Le, 2011, « Sociologie de l'État en recomposition », Revue francaise de sociologie, 52, 3, p. 453480.

KNORR-CETINA Karin D., 2012, "Financial analysis: Epistemic profile of an evaluative science », in Michele Lamont, Charles Camic, et Neill Gross (dir.), Social knowledge in the making.

KRIMSKY Sheldon, 2004, Science in the Private Interest: Has the Lure of Profits Corrupted Biomedical Research?, Rowman \& Littlefield.

LAVE R., MIROWSKI P., et RANDALLS S., 2010, «Introduction: STS and Neoliberal Science », Social Studies of Science, 40, 5, p. 659675.

MICHAELS David (dir.), 2008, Doubt is Their Product: How industry's assault on science threatens your health, Oxford, New York, Oxford University Press.

MOGHISSI A. Alan, STRAJA Sorin R., LOVE Betty R., BRIDE Dennis K., et STOUGH Roger R., 2014, «Innovation in regulatory science: evolution of a new scientific discipline », Technology \& Innovation, 16, 2, p. 155-165.

ORESKES Naomi et CONWAY Erik M., 2010, Merchants of Doubt: How a Handful of Scientists Obscured the Truth on Issues from Tobacco Smoke to Global Warming, Bloomsbury Publishing USA.

OTA, 1983, The Information Content of Premanufacture Notices. Background paper. Congress of the United States, Office of Technology Assessment.

SHACKLEY Steven et WYNNE Brian, 1995, «Global climate change: the mutual construction of an emergent science-policy domain », Science and Public Policy, 22, 4, p. 218230.

STRANGE Susan, 2015, States and Markets, Bloomsbury Publishing.

VARENNE Franck et SILBERSTEIN Marc, 2013, Modéliser \& simuler. Epistémologies et pratiques de la modélisation et de la simulation, tome 1, Editions Matériologiques.

VOGEL David, 1988, « The" new" social regulation in historical and comparative perspective », in Lawrence M. Friedman et Harry N. Scheiber (dir.), American Law and the Constitutional Order: Historical Perspectives, Cambridge, MA, London, Harvard University Press, p. 431448. 
VOGEL Sarah A. et ROBERTS Jody A., 2011, « Why the toxic substances control act needs an overhaul, and how to strengthen oversight of chemicals in the interim », Health affairs, 30,5 , p. 898-905.

WENGLE Susanne, 2016, « When experimentalist governance meets science-based regulations; the case of food safety regulations », Regulation \& Governance, 10, 3, p. 262 283.

ZEEMAN M, NABHOLZ JV and CLEMENTS RG, 1993, The Development of SAR/QSAR for Use Under EPA's Toxic Substances Control Act (TSCA): An Introduction. In: Gorsuch J, Dwyer J, Ingersoll C, et al. (eds), Environmental Toxicology and Risk Assessment - 2nd Volume, Philadelphia: American Society for Testing and Materials, p. 523-539. 